\title{
EL IMPACTO DE LA ESTRUCTURA DE LAS REDES SOCIALES SOBRE EL ACCESO DE LOS INDIVIDUOS AL MERCADO LABORAL
}

\section{THE EFFECT OF SOCIAL NETWORKS STRUCTURE ON THE INDIVIDUALS' ACCESS TO THE LABOUR MARKET}

\author{
JosÉ IGNACIO GARCíA-VALDECASAS joseignacio.garcia-valdecasas@uc3m.es \\ Universidad Carlos III de Madrid. España.
}

\begin{abstract}
RESUMEN
Este trabajo pretende analizar el efecto de la estructura de las redes sociales sobre el acceso de los individuos al mercado laboral a través de la difusión de la información que tiene lugar en dichas redes (un aspecto del capital social). Para realizar tal análisis, se ha llevado a cabo una serie de experimentos virtuales con redes artificiales, utilizando para ello la simulación basada en agentes y el análisis de redes sociales. Los resultados muestran que no solo el tipo de estructura (mundos pequeños, redes de libre escala, redes aleatorias y redes regulares), sino también ciertas propiedades estructurales como la densidad de red, el índice de globalización de vínculos o el grado de desigualdad en la distribución de vínculos influyen considerablemente sobre las oportunidades de los agentes de conseguir empleo.
\end{abstract}

\section{Palabras Clave}

Análisis de redes sociales; Capital social; Experimentos virtuales; Redes artificiales; Simulación basada en agentes.

\section{Abstract}

This work attempts to analyse the effect of social networks structure on the access to the labour market for individuals through the diffusion of information which takes place in such networks (an aspect of social capital). For that purpose, it combines agent-based modelling and social network analysis in order to carry out virtual experiments with artificial networks. The results show that not only the type of structure (small world, free scale networks, random networks and regular networks), but also certain structural properties such as the density of network, the index of globalization in links or the degree of inequality in the distribution of links affect deeply on the opportunities of agents in order to achieve employment.

\section{KEYWORDS}

Agent-Based Modelling; Artificial Networks; Social Capital; Social Network Analysis; Virtual Experiments. 


\section{INTRODUCCIÓN}

Numerosas investigaciones tanto teóricas como empíricas han analizado el efecto de las redes sociales sobre el acceso de los individuos al mercado laboral (Beaman 2012; Burt 2004 y 2005; Calvó-Armengol 2004 y 2006; Calvó-Armengol y Jackson 2004 y 2007; Franzen y Hangartner 2006; García Faroldi 2007; Granovetter 1973 y 1974; López-Roldan y Alcaide 2011; Montgomery 1991; Requena 1991 y 2006; Toledo y Bastourre 2006). Sin embargo, hasta el momento no se han realizado suficientes investigaciones sobre el impacto de la estructura de las redes sociales sobre dicho acceso al mundo laboral. Este déficit de investigaciones se debe a la dificultad o imposibilidad de conseguir datos empíricos suficientes para analizar cómo la estructura de las redes, por la que puede fluir información relevante sobre puestos de trabajo, afecta a tal acceso. Para suplir esta falta de datos, esta investigación combina la simulación basada en agentes (Agent-Based Modelling) (Axelrod 1997; Epstein 2006; García-Valdecasas 2011b; Gilbert 2008; Gilbert y Abbott 2005; Gilbert y Troitzsch 2005; González-Bailón 2004; Macy y Flache 2009; Macy y Miller 2002; Squazzoni 2012) y el análisis de redes sociales (Social Network Analysis) (Boccaletti et al. 2006; Carrington et al. 2005; Hanneman y Riddle 2005; Molina 2001; Moody 2009; Requena 1989 y 2003; Rodríguez 1995; Wasserman y Faust 1994) para llevar a cabo experimentos virtuales con redes artificiales con el objetivo de analizar las repercusiones de la estructura de las redes sociales sobre las oportunidades de los individuos para conseguir empleo.

Es importante realizar una serie de consideraciones sobre la metodología utilizada en esta investigación y sobre el concepto de capital social, entendido como aquellos recursos que los individuos pueden obtener de las redes sociales por estar insertos en ellas. Respecto a la metodología se debe subrayar, en primer lugar, que los experimentos virtuales, obviamente, no pueden proporcionar datos empíricos y, por tanto, no pueden sustituir a la investigación empírica. Sin embargo, los resultados de dichos experimentos pueden generar diferentes hipótesis empíricas que posteriormente pueden contrastarse, así como sugerir diversas cuestiones teóricas que pueden enriquecer nuestra visión sobre los fenómenos sociales. Es decir, la combinación de la simulación basada en agentes y el análisis de redes sociales puede servir de guía a la investigación de la misma manera que lo hace normalmente la teoría informal discursiva (la teoría sociológica textual).

Otra consideración clave es que las redes artificiales utilizadas en este trabajo de investigación tratan de ser modelos de las redes reales, esto es, que las primeras deben de representar en algún sentido tanto la dinámica como la estructura de las segundas. Si esto es así, entonces los resultados de los experimentos virtuales con las redes artificiales deberían ser semejantes a los resultados arrojados por la investigación empírica con las redes reales. En este caso, las conclusiones de los experimentos virtuales con redes artificiales podrían servir de guía a ciertas políticas sociales con objeto de facilitar el acceso de los individuos al mundo laboral. 
Conviene también comentar que la revolución en el campo de la informática ha provocado, en los últimos treinta años, cambios importantes en la manera de realizar investigación social. Los sociólogos normalmente emplean los ordenadores para estimar modelos estadísticos a partir de datos empíricos o para buscar soluciones analíticas a sistemas de ecuaciones que representan fenómenos sociales. Sin embargo, desde hace dos décadas, los científicos sociales han empezado a utilizar con profusión los ordenadores para simular las interacciones entre individuos insertos en estructuras sociales (como las redes sociales) y analizar las consecuencias de dichas interacciones (Axelrod 1997; Epstein 2006; Gilbert y Abbott 2005; Squazzoni 2012). Este trabajo de investigación pertenece a esta última línea de investigación.

En segundo lugar, respecto al capital social se debe indicar que el presupuesto de partida de esta investigación es sumamente simple: la estructura de las redes sociales es importante (García-Valdecasas 2011a). La estructura de las redes puede generar capital social y el capital social puede producir tanto beneficios públicos como privados. Dos de los aspectos más relevantes del capital social son la información que fluye por las redes sociales (Burt 1992; 2004 y 2005; Granovetter 1973 y 1995) y las obligaciones de reciprocidad entretejidas en dichas redes sociales (Herreros 2002; Coleman 1990; Bourdieu 1986). Esta investigación, en particular, se centra en el flujo de información sobre ofertas de empleo que puede recorrer las estructuras de las redes sociales y así facilitar el acceso de los individuos al mercado laboral.

Este trabajo parte, pues, de una definición de capital social que vincula la estructura de las redes sociales donde los individuos están insertos con los recursos disponibles para dichos individuos en tales redes (Lin 2001; Coleman 1988 y 1990; Bourdieu 1986). El capital social, tal como aquí se define, consiste en una serie de recursos que los individuos pueden obtener a partir de las estructuras de las redes. Los sujetos no pueden acceder a dichos recursos de capital social si no participan de alguna manera en tales redes sociales. Así pues, un individuo aislado que no participe en ninguna red social no podrá disfrutar de los recursos del capital social.

El artículo está organizado de la siguiente forma: en primer lugar, se describen las redes artificiales creadas en este trabajo de investigación para realizar los experimentos virtuales. En segundo lugar, se investiga el efecto de diferentes tipos de estructuras de red (regular, aleatoria, mundo pequeño y escala libre) sobre el acceso de los agentes al mercado laboral. Por último, se analiza el impacto de distintas propiedades estructurales (densidad de red, índice de globalización de vínculos y grado de desigualdad en la distribución de vínculos) sobre dicho acceso al mundo laboral. En resumen, se trata no solo de analizar el impacto de diferentes tipos de estructuras de redes y distintas propiedades estructurales sobre las oportunidades de los agentes de encontrar trabajo, sino también de mostrar la utilidad de la combinación de la simulación basada en agentes y el análisis de redes en la investigación social. 


\section{LAS REDES ARTIFICIALES}

Las redes artificiales utilizadas en los experimentos virtuales de esta investigación han sido creadas con el entorno de programación NetLogo (Wilensky 1999). El punto de partida es un conjunto de 100 redes personales aisladas entre sí, constituidas cada una de ellas por un agente principal unido a varios agentes secundarios a través de vínculos fuertes. El tamaño de dichas redes está basado en los datos empíricos proporcionados por Hill y Dunbar (2003). La Tabla I muestra la distribución de los tamaños de las redes personales, pero por razones computacionales se han reducido por un factor de 10, manteniendo constantes los porcentajes. Así pues, en estas redes personales, el número de agentes secundarios enlazados a cada agente principal varía entre 0 y 40 .

Tabla I.

Tamaño de las redes personales.

\begin{tabular}{c|c}
\hline Número de vínculos fuertes del agente principal & $\%$ \\
\hline $0-5$ & 7,0 \\
$6-10$ & 18,6 \\
$11-15$ & 32,6 \\
$16-20$ & 16,3 \\
$21-25$ & 9,3 \\
$26-30$ & 9,3 \\
$31-35$ & 4,6 \\
$36-40$ & 2,3 \\
Total & 100 \\
\hline
\end{tabular}

Elaboración propia a partir de los datos de Hill y Dunbar (2003).

Sin embargo, las redes artificiales utilizadas en esta investigación necesitan una estructura global y no solo un conjunto de 100 redes personales aisladas entre sí (González-Bailón 2006). Para ello, se colocan al azar a los 100 agentes principales con sus respectivos agentes secundarios en un "torus" (uno de los mundos posibles de NetLogo). Luego, cada agente secundario se enlaza a través de vínculos fuertes a todos los agentes situados dentro del área de un círculo cuyo radio es $\alpha$ y cuyo centro es dicho agente, y a continuación se reemplazan, con una probabilidad $\beta$, los vínculos fuertes de cada agente con los vecinos por $\gamma$ vínculos débiles (puentes estructurales) con agentes situados más allá del vecindario. El parámetro $\beta$, por tanto, puede ser considerado como un índice de globalización de los vínculos de una red. Que un vínculo fuerte y local pueda ser sustituido por más de un vínculo débil y global (cuando $\gamma>1$ ) puede ser justificado diciendo que los recursos en la creación y mantenimiento de vínculos débiles son mucho menores que los que requieren los vínculos fuertes (Burt 2004 y 2005; Granovetter 1973 y 1974). 
Conviene subrayar que las redes artificiales utilizadas en estos experimentos virtuales distinguen entre vínculos fuertes y débiles (Burt 2004 y 2005; Granovetter 1973 y 1974). Las personas suelen estar rodeadas de un núcleo de vínculos fuertes formado por un número pequeño de personas con las que se mantiene un contacto estrecho y frecuente, como la familia o los amigos. Junto a este círculo fuerte existe una gran cantidad de vínculos débiles con otras personas con las que el contacto es más superficial y menos frecuente, que pueden ser conocidos, vecinos o colegas. Granovetter (1973: 1361) indicó que la fuerza de un vínculo es una combinación lineal de la cantidad de tiempo, la intensidad emocional, la ayuda recíproca y la intimidad mutua que caracteriza al vínculo.

Sin embargo, del hecho de que el vínculo entre dos conocidos sea débil no se puede derivar en absoluto que dicho vínculo proporcione menos recursos. Los vínculos débiles pueden proporcionar, por ejemplo, información más diversificada sobre ofertas de trabajo que los vínculos fuertes, que suelen transmitir información redundante (Burt 2004 y 2005; Granovetter 1973 y 1974). Es decir, las redes de vínculos débiles no solo pueden proporcionar más información por el hecho de que son más grandes, sino que la relevancia de la información transmitida puede ser mayor. Como señaló en su día Granovetter (1973: 1366) "cualquier información difundida puede llegar a un número mayor de gente y recorrer una mayor distancia social [...] cuando se utilizan vínculos débiles que cuando de usan vínculos fuertes". Los amigos de un individuo pueden poseer información parecida a la de dicho individuo, que obtiene, por tanto, información redundante de ellos; en cambio, alguien que sea solo un conocido puede aportar información novedosa (Lin 2001: 67). Por ejemplo, la participación en una asociación puede suministrar información relevante sobre temas alejados de los objetivos concretos de la asociación: al formar parte de una asociación de ornitología se puede obtener sin duda información sobre pájaros y disfrutar con los compañeros de una salida al campo, pero también el compañero de al lado - que es economista- puede proporcionar información pertinente sobre las ofertas de empleo en la empresa donde trabaja (Herreros 2002).

En estas redes artificiales se ha considerado que la velocidad con que se transmite la información a través de los vínculos fuertes - aunque pueda ser redundante- es $\delta$ veces más rápida que mediante los vínculos débiles (siendo $\delta>1$ ). Este presupuesto se puede justificar indicando que el contacto entre los agentes a través de los vínculos fuertes es más frecuente que mediante los vínculos débiles (Burt 2004 y 2005; Granovetter 1973 y 1974).

Se puede modificar la densidad de red variando los parámetros $\alpha$ y $\gamma$. Asimismo, se puede cambiar el tipo de estructura modificando el factor $\beta$ : si $\beta=0$, las redes artificiales tienen una estructura básicamente regular, y si $\beta=1$, poseen una estructura fundamentalmente aleatoria. Dicho con otras palabras, cuando $\beta=0$, ningún vínculo local es reemplazado y la red regular inicial no se modifica, mientras que cuando $\beta=1$, todos los vínculos locales son reemplazados por vínculos globales y se genera una red aleatoria. Sin embargo, cuando los valores de $\beta$ están comprendidos entre 0 y 1 , es decir, $0<\beta<1$, las redes artificiales pueden ser consideradas como mundos pequeños (Watts 1999a; 1999b y 2003; Watts y Strogatz 1998). Así pues, se pueden imaginar los mundos pequeños como estructuras intermedias entre las redes regulares y las aleato- 
rias. Este procedimiento en la creación de mundos pequeños artificiales en el ordenador permite representar los dos rasgos más sobresalientes de los mundos pequeños reales: la cohesión local (a través de vínculos fuertes y locales) y la conexión global (debido a los vínculos débiles y globales).

Además de las redes regulares, aleatorias y mundos pequeños, estos experimentos virtuales utilizan redes de escala libre (Barabási 2002; Barabási y Bonabeau 2003). Para crear en el ordenador este tipo de estructuras se utiliza un mecanismo basado en dos conceptos: el crecimiento y la conexión preferencial (Barabási y Albert 1999). Ambos rasgos pueden observarse en las redes sociales reales: el crecimiento hace referencia a que las redes poseen una cantidad creciente de agentes, y la conexión preferencial hace alusión a que los nuevos agentes que llegan a la red se conectan con mayor probabilidad a aquellos agentes más conectados. La estructura empieza con una red personal - como las descritas anteriormente- situada en el mundo de NetLogo. Posteriormente, se añade una segunda red personal en la que se seleccionan $\varepsilon$ agentes que se conectan con cualquier agente de la primera red personal mediante vínculos débiles con una probabilidad proporcional al número de vínculos que poseen dichos agentes de la primera red. A continuación, en cada paso de tiempo, se añade una nueva red personal (hasta llegar a las 100 redes personales), y se sigue el procedimiento anterior; es decir, los nuevos agentes se unen preferencialmente con aquellos agentes más conectados. Así pues, un agente que tenga el doble número de vínculos que otro poseerá el doble de probabilidad de recibir un nuevo vínculo. De esta forma, este procedimiento recoge el hecho de que los agentes más antiguos son los que tienen una mayor probabilidad de estar más conectados. Un aspecto notable de este algoritmo es que se puede modificar el grado de desigualdad en la distribución de vínculos entre los agentes de la red variando el parámetro $\varepsilon$ de dicha red.

Posteriormente, con objeto de conocer los valores de las propiedades estructurales de las redes artificiales creadas, se pueden exportar dichas redes al programa de análisis de redes Ucinet-Netdraw. Además del índice de globalización de vínculos comentado más arriba, las propiedades estructurales que se van a utilizar en estos experimentos virtuales son la densidad de red, la longitud media de paso y el índice de Gini de la distribución de vínculos.

La primera propiedad, la densidad de red $(D)$, es una medida del grado de conectividad de la red, y se puede definir como el cociente entre el número de vínculos existentes y el número de vínculos posibles de la red (Carrington, Scott y Wasserman 2005; Diestel 2000; Wasserman y Faust 1994). Cuanto mayor sea el número de vínculos existentes en una red, mayor será la densidad. Se puede calcular mediante la siguiente fórmula:

$$
D=\frac{2 m}{n(n-1)}
$$

siendo $m$ el número total de vínculos de la red y $n$ el número de agentes de dicha red. 
La segunda propiedad, la longitud media de paso de red $(\bar{L})$, es una medida del grado de cercanía entre los agentes de una red (Carrington, Scott y Wasserman 2005; Diestel 2000; Wasserman y Faust 1994). Cuanto menor sea la longitud media de paso, mayor será la cercanía entre los agentes, y, por tanto, más rápidamente podrá difundirse la información en la red. Es importante subrayar que cuanto más rápida sea la velocidad de difusión de la información en la red, mayor acceso tendrán los agentes de dicha red al mercado de trabajo (Burt 2004 y 2005). Por velocidad de difusión de la información se entiende, en el caso de este artículo, el tiempo que se tarda en que todos los agentes de la red sean informados de una oferta de empleo. Por consiguiente, todo factor que reduzca la longitud media de paso aumenta la velocidad de difusión y facilita el acceso de los agentes al mercado de trabajo; por el contrario, todo factor que aumente la longitud media de paso, reduce la velocidad de difusión y dificulta la entrada al mundo laboral.

La longitud media de paso de un agente $i$, que se nota por $L_{i}$, es la media de todas las longitudes de paso entre el agente $i$ y el resto de los agentes. Se determina a través de la siguiente fórmula:

$$
L_{i}=\frac{\sum_{i \neq j} l_{i, j}}{(n-1)}
$$

siendo $n$ el número de agentes de la red y $l_{i, j}$ la longitud de paso entre dos agentes $i$ y $j$, es decir, la distancia geodésica entre dichos agentes, esto es, el número de vínculos entre tales agentes por el camino más corto. La longitud media de paso de red $(L)$ es la media de las longitudes media de paso de todos los agentes:

$$
\bar{L}=\frac{\sum_{i} L_{i}}{n} .
$$

El acceso de los agentes al mercado laboral depende de muchos factores entre los cuales cabe destacar la velocidad de difusión de la información de las ofertas de trabajo por las redes. Sin embargo, el modelo de simulación permite mantener constantes todos los factores excepto la velocidad de difusión de la información para analizar cómo influye esta en el acceso de los agentes al mercado laboral. Así pues, los experimentos virtuales permiten aplicar la cláusula ceteris paribus para centrarnos en cómo la velocidad de difusión influye en el acceso al mundo del trabajo.

Por último, en este trabajo se utiliza el índice de Gini $(G)$ como medida del grado de desigualdad en la distribución de vínculos entre los agentes de una red (Allison 1978). 
Dicho índice está relacionado con la desigualdad social (Coleman 1988; Lin 2001), y se puede calcular haciendo uso de la siguiente fórmula:

$$
\mathrm{G}=\frac{\frac{1}{n^{2}} \sum_{i=1}^{n} \sum_{j=1}^{n}\left|l_{i}-l_{j}\right|}{2 \mu}
$$

donde $\mu$ es la media de la distribución del número de vínculos de los agentes de una red, $n$ es el número total de agentes de la red $y I_{i}$ es el grado modal del agente $i$, es decir, el número de vínculos del agente $i$.

En resumen, las redes artificiales creadas para estos experimentos virtuales están caracterizadas por 5 parámetros: $\alpha, \beta, \gamma, \delta, \varepsilon$. El parámetro $\alpha$ es el radio del círculo de vecinos de los agentes secundarios; $\gamma$ es el número de vínculos débiles y globales que puede sustituir a un vínculo fuerte y local; tanto $\alpha$ como $\gamma$ están relacionados con la densidad de red, $D ; \beta$ (el índice de globalización de vínculos) es la probabilidad de cambiar vínculos fuertes y locales con los vecinos por $\gamma$ vínculos débiles y globales con agentes situados más allá del vecindario, y tiene que ver con el tipo de estructura de red (regular, mundo pequeño y aleatoria); $\delta$ es el número de veces que la velocidad en la transmisión de la información es más rápida entre los vínculos fuertes que entre los débiles; $y$, por último, $\varepsilon$ es el número de agentes de una red personal que se conectan con otros agentes en una red de libre escala, y está ligado al índice de Gini del grado de desigualdad en la distribución de vínculos entre los agentes, $G$.

\section{EXPERIMENTOS VIRTUALES}

Se han realizado cuatro tipos diferentes de experimentos virtuales con redes artificiales, y cada uno de ellos se ha ejecutado 100 veces. El espacio paramétrico de cada experimento se ha especificado debajo del título del gráfico correspondiente. Lo importante de estos experimentos no son los resultados numéricos concretos, que dependen de los valores arbitrarios de los parámetros iniciales, sino la forma geométrica de las funciones, que se mantiene constante e independiente de los valores concretos de los parámetros de partida.

\section{Impacto de diferentes estructuras de red en la velocidad de difusión de la información}

Para analizar cómo la estructura de red afecta a la velocidad de difusión de la información en las redes y, por tanto, al acceso de los agentes al mercado laboral, se puede realizar el siguiente experimento virtual: cada agente de la red posee un atributo con dos posibles valores: 00 1. "0" representa el desconocimiento de una oferta de trabajo y, por el contrario, "1" el conocimiento de dicha oferta. Inicialmente todos los agentes de la red poseen el atributo de valor 0 , y solo un único agente, elegido al azar, tiene el atributo 
de valor 1. Luego este agente transmite la información a los agentes vinculados a él en una iteración o paso de tiempo. Después estos agentes transmiten la información a los agentes vinculados a ellos en una segunda iteración, y así sucesivamente hasta que toda la población de la red tenga el atributo 1 (véase González-Bailón 2006).

En el experimento se crean tres redes con diferentes estructuras (con distintos valores de $\beta$ ), pero con la misma densidad de red $(D=0,15)$, y, posteriormente, se investiga el número de pasos tiempo o iteraciones necesarios para que la información sobre la oferta de trabajo llegue a toda la población. Los resultados se pueden observar en el gráfico 1.

Gráfico 1.

Velocidad de difusión de la información en diferentes estructuras de red.

$$
(\alpha=2 ; \beta=0,0,3,1 ; \gamma=1 ; \delta=4) \text {. }
$$

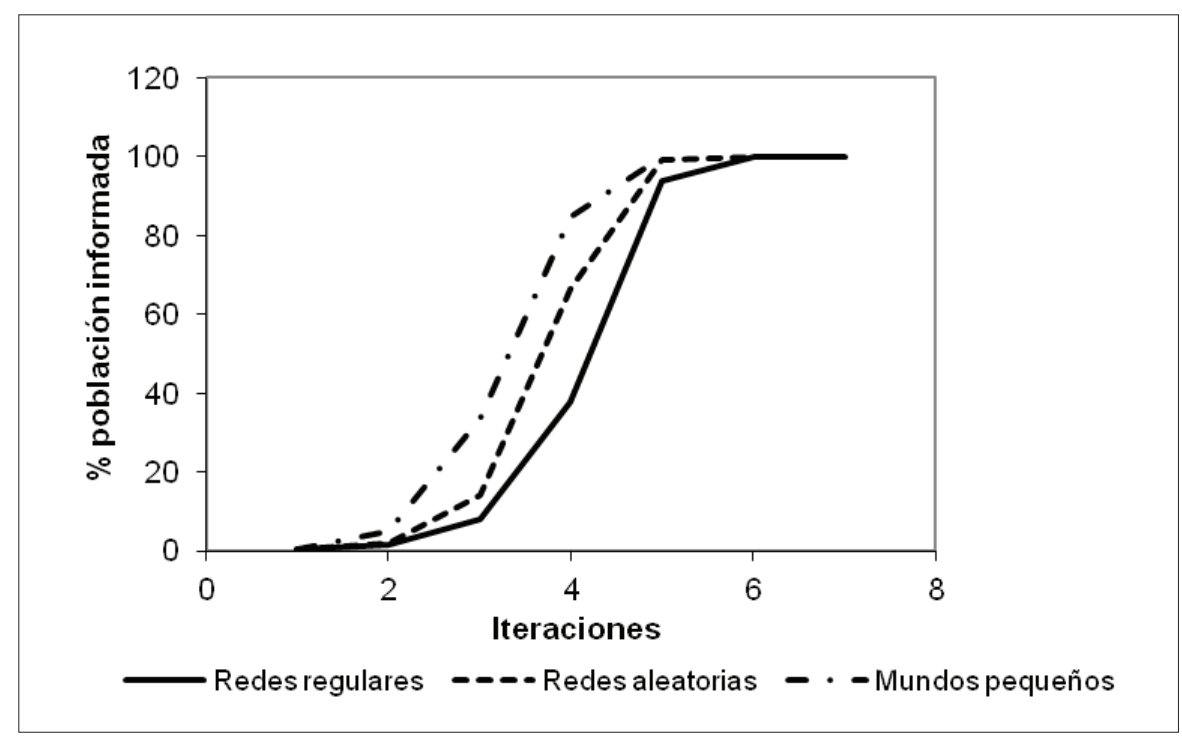

Fuente: Elaboración propia.

Como muestra el gráfico anterior, los mundos pequeños son las estructuras que necesitan menor número de iteraciones para que un mismo porcentaje de la población conozca la oferta de trabajo. En concreto, los mundos pequeños $(\beta=0.3)$ necesitan tan solo 5 iteraciones para que el $100 \%$ de la población tenga conocimiento de la oferta; sin embargo, las redes aleatorias $(\beta=1)$ y las redes regulares $(\beta=0)$ necesitan 6 y 7 iteraciones, respectivamente. Es decir, si el número de personas informadas es el mismo, los mundos pequeños requieren menos tiempo para la difusión de la información de una oferta de trabajo que las redes aleatorias, y estas, a su vez, son más rápidas en la difusión de la 
información que las redes regulares; y si la velocidad de difusión de la información es mayor en los mundos pequeños, ceteris paribus, los individuos de dichos mundos tienen mayores posibilidades de conseguir antes un puesto de trabajo.

De igual manera, para un mismo número de iteraciones, como indica el gráfico anterior, el porcentaje de población con conocimiento de la oferta de trabajo es mayor en los mundos pequeños que en el resto de las redes. Es decir, para el mismo intervalo de tiempo, el número de agentes enterados de la oferta es mayor en los mundos pequeños que en el resto de las estructuras. Por ejemplo, si el número de pasos de tiempo es 4, el porcentaje de la población con conocimiento de la oferta es del $90 \%$ en los mundos pequeños, $70 \%$ en las redes aleatorias y $40 \%$ en las redes regulares.

En resumen, dos conclusiones pueden ser extraídas de esta primera serie de experimentos: en primer lugar, la importancia de la estructura de red en la velocidad de difusión de la información sobre ofertas de empleo y, por consiguiente, en el acceso de los agentes al mercado laboral; $y$, en segundo lugar, la relevancia de los mundos pequeños en dicha velocidad de difusión de la información. Así pues, los agentes de los mundos pequeños tienen más probabilidades de conseguir antes un empleo que los agentes de las redes aleatorias o regulares. Quizás los mundos pequeños hayan sobrevivido desde un punto de vista evolutivo porque confieren más prestaciones que el resto de las estructuras en el mismo intervalo de tiempo.

\section{Efecto de la densidad sobre la longitud de paso en distintas estructuras de redes}

La segunda tanda de experimentos virtuales analiza cómo influye la densidad de red (D) en la longitud media de paso $(L)$ en diferentes estructuras de redes, y, por tanto, en la velocidad de la difusión de la información en dichas estructuras.

Como se muestra en el gráfico 2, a medida que aumenta la densidad de red disminuye la longitud media de paso en los diferentes tipos de estructuras de redes; y si se reduce la longitud media de paso, se incrementa la velocidad de la difusión de la información que pasa por dichas estructuras, es decir, aumenta el acceso de los agentes al mundo laboral.

Se puede aplicar este resultado al crecimiento explosivo de las redes sociales virtuales (Facebook o Twitter, entre otras muchas). Dicho crecimiento conlleva un aumento considerablemente del número de vínculos débiles entre los individuos, y, por consiguiente, un incremento en la densidad de red. Lo anterior implica, como pone de manifiesto el gráfico 2, una reducción en la longitud media de paso, y, por tanto, un incremento en la velocidad de la difusión de la información. Es decir, las "cyber social networks" aumentan las posibilidades de los individuos para encontrar empleo (Lin 2001).

También se puede observar en el gráfico anterior qué tipo de estructura permite una mayor difusión de la información, y, por consiguiente, cuál genera un mayor acceso de los agentes al mercado de trabajo: para una misma densidad, la longitud media de paso de las redes regulares $(\beta=0)$ es mayor que la de las redes aleatorias $(\beta=1)$, siendo esta a su vez mayor que la longitud media de paso de los mundos pequeños $(\beta=0,3)$. 


\section{Gráfico 2.}

Impacto de la densidad sobre la longitud de paso en distintas estructuras de redes.

$$
(\alpha=2 ; \beta=0,0,3,1 ; \gamma=2,3,4,5,6 ; \delta=4) \text {. }
$$

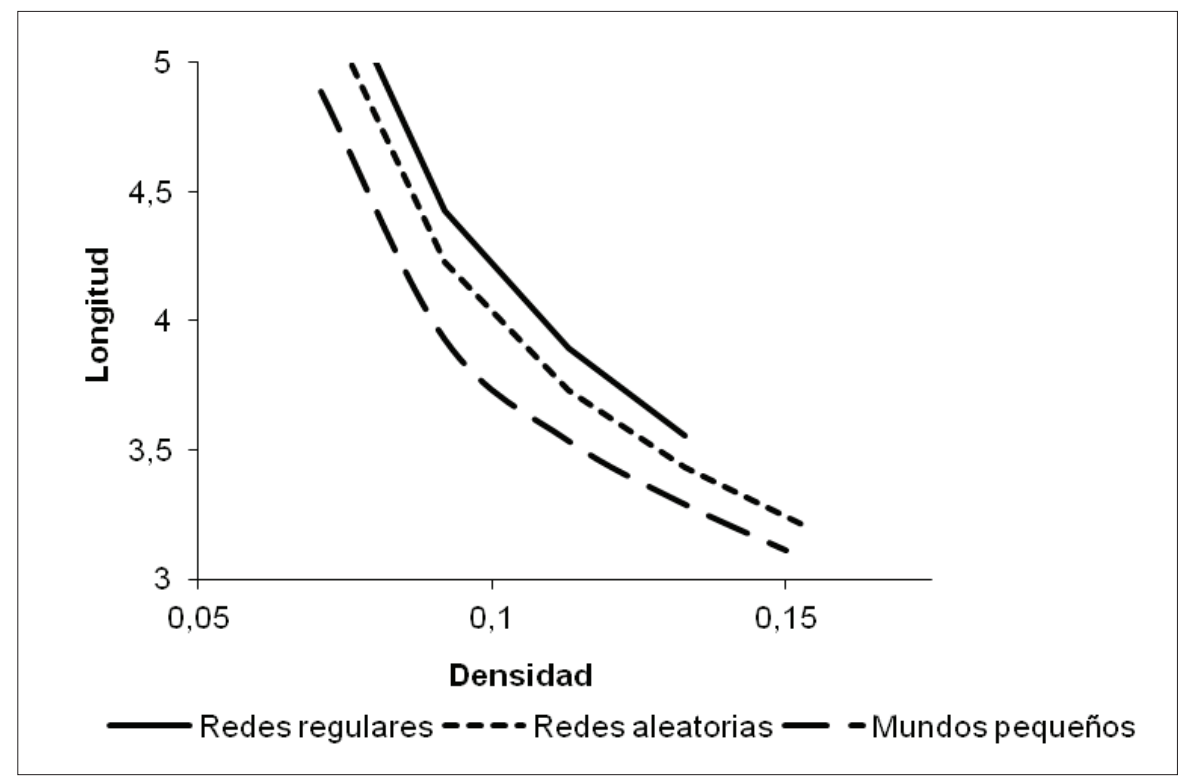

Fuente: Elaboración propia.

Esto quiere decir que una red regular es menos eficiente en la transmisión de la información que una red aleatoria; y una red aleatoria, a su vez, es menos eficaz que un mundo pequeño. Es decir, se puede mostrar de nuevo que los mundos pequeños son mucho más eficaces en la transmisión de la información que otros tipos de estructuras. Así pues, los agentes de los mundos pequeños, ceteris paribus, tienen más posibilidades de encontrar trabajo que los de las redes aleatorias, y los de las redes aleatorias poseen más posibilidades de conseguir empleo que los de las redes regulares.

Asimismo, para una misma longitud media de paso, la densidad de los mundos pequeños es menor que la de las redes aleatorias, y éstas, a su vez, tienen una menor densidad que la de las redes regulares. Por lo tanto, si las tres redes son iguales respecto a la transmisión de la información, los mundos pequeños son de menor coste que las redes aleatorias, y dichas redes, a su vez, son más "económicas" que las regulares porque poseen menos densidad de red. Es decir, las tres redes pueden tener las mismas prestaciones, pero los mundos pequeños requieren menor esfuerzo en su construcción y mantenimiento que las redes aleatorias; y estas, a su vez, suponen menor esfuerzo que las regulares. 
En resumen, los resultados de la segunda tanda de experimentos confirman, como sugieren algunas investigaciones, que un aumento en el número de vínculos débiles (Granovetter 1973 y 1974) o de puentes estructurales (Burt 2004 y 2005) genera más oportunidades entre los agentes de una red de encontrar empleo (Lin 2001). Además, dichos resultados vuelven a poner de manifiesto que existen ciertas estructuras, como los mundos pequeños, que favorecen más el acceso de los agentes al mercado de trabajo que otras.

\section{Impacto del índice de globalización sobre la longitud media de paso}

La tercera serie de experimentos virtuales analiza cómo el índice de globalización de los vínculos de red $(\beta)$ afecta a la longitud media de paso de dicha red $(L)$ para una densidad determinada. Cuanto menor sea la longitud media de paso, mayor será la velocidad de la difusión de la información, y, por tanto, mayor probabilidad tendrán los agentes de dicha red de encontrar empleo.

El gráfico 3 muestra que, para una misma densidad $(D=0,15)$, existe un valor del índice de globalización $(\beta=0,6)$ para el cual la longitud media de paso es mínima, y, por consiguiente, la velocidad de difusión de la información que atraviesa la red es máxima.

Gráfico 3.

Efecto del índice de globalización sobre la longitud media de paso. $(\alpha=2 ; \beta=0,0,1,0,2,0,3,0,4,0,5,0,6,0,7,0,8,0,9,1 ; \gamma=1 ; \delta=4)$.

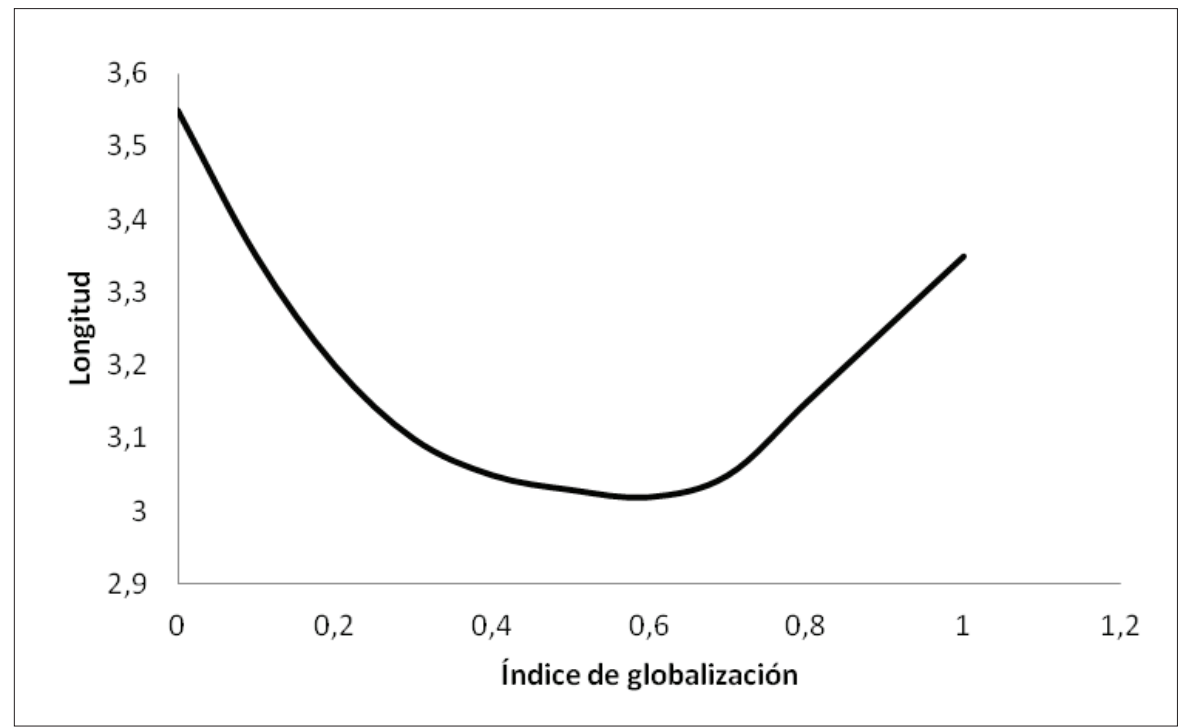

Fuente: Elaboración propia. 
Existe, por tanto, un límite físico que no se puede superar en la velocidad de la difusión de la información, es decir, existe un índice de globalización óptimo para encontrar trabajo. Nótese que la mayoría de los mundos pequeños $(0<\beta<1)$ poseen menores longitud media de paso que las redes regulares $(\beta=0)$ y las redes aleatorias $(\beta=1), y$, dentro de los mundos pequeños, aquel caracterizado por un valor de $\beta=0,6$ es el que tiene la menor longitud media de paso, y, por tanto, el que brinda a los agentes un mayor acceso al mercado de trabajo.

Así pues, las redes locales caracterizadas por valores de $\beta$ bajos y por numerosos vínculos fuertes, así como las redes globales caracterizadas por valores de $\beta$ altos y por cuantiosos vínculos débiles entre los agentes generan menos oportunidades para dichos agentes de acceder a puestos de trabajo que las redes intermedias con valores de $\beta$ moderados que muestran una combinación equilibrada entre vínculos fuertes y débiles para una misma densidad de red.

En resumen, la tercera serie de experimentos muestra la existencia de un valor de $\beta$ óptimo para el acceso de los agentes al mercado laboral. Los individuos de sociedades muy cerradas constituidas por redes locales y los de sociedades muy abiertas formadas por redes globales tienen menos acceso al mercado de trabajo, según estos resultados, que los individuos que pertenecen a sociedades situadas en un punto intermedio entre ambos extremos para un mismo valor de densidad.

\section{Efecto del grado de desigualdad en la distribución de vínculos sobre la longitud media de paso}

En la última tanda de experimentos virtuales se analiza cómo el índice de Gini de la desigualdad de la distribución de vínculos $(G)$ entre los agentes de una red de libre escala influye en la longitud media de paso de dicha red $(\bar{L})$ para una densidad determinada. Cuanto menor sea la longitud media de paso, mayor será la velocidad de difusión de la información, y, por consiguiente, mayor acceso tendrán los agentes de dicha red al mercado de trabajo.

Como se observa en el gráfico 4 , para una misma densidad $(D=0,15)$, existe un índice de Gini (G = $39 \%$ ) para el cual la longitud media de paso es mínima, y, por tanto, la velocidad del flujo de información que recorre la red es máxima, es decir, existe un índice de Gini óptimo para encontrar empleo. Por lo tanto, la velocidad en la transmisión de la información para índices de Gini superiores o inferiores a dicho óptimo es siempre menor, y, por consiguiente, el acceso al mercado de trabajo por parte de los agentes es también menor.

Así pues, redes desigualitarias con índices de Gini altos (extremo derecho del gráfico 4) o redes igualitarias con índices de Gini bajos (extremo izquierdo del gráfico 4) generan menos oportunidades de acceder a puestos de trabajo para los agentes que redes con índices de Gini de valores intermedios. Es decir, los resultados de esta investigación sugieren que redes con niveles intermedios de desigualdad son más proclives a generar mayores oportunidades para acceder al mercado de trabajo. 
Gráfico 4.

Impacto del índice de Gini sobre la longitud de paso en redes de escala libre.

$(\delta=5 ; \varepsilon=1,2,4,8,16,32)$.

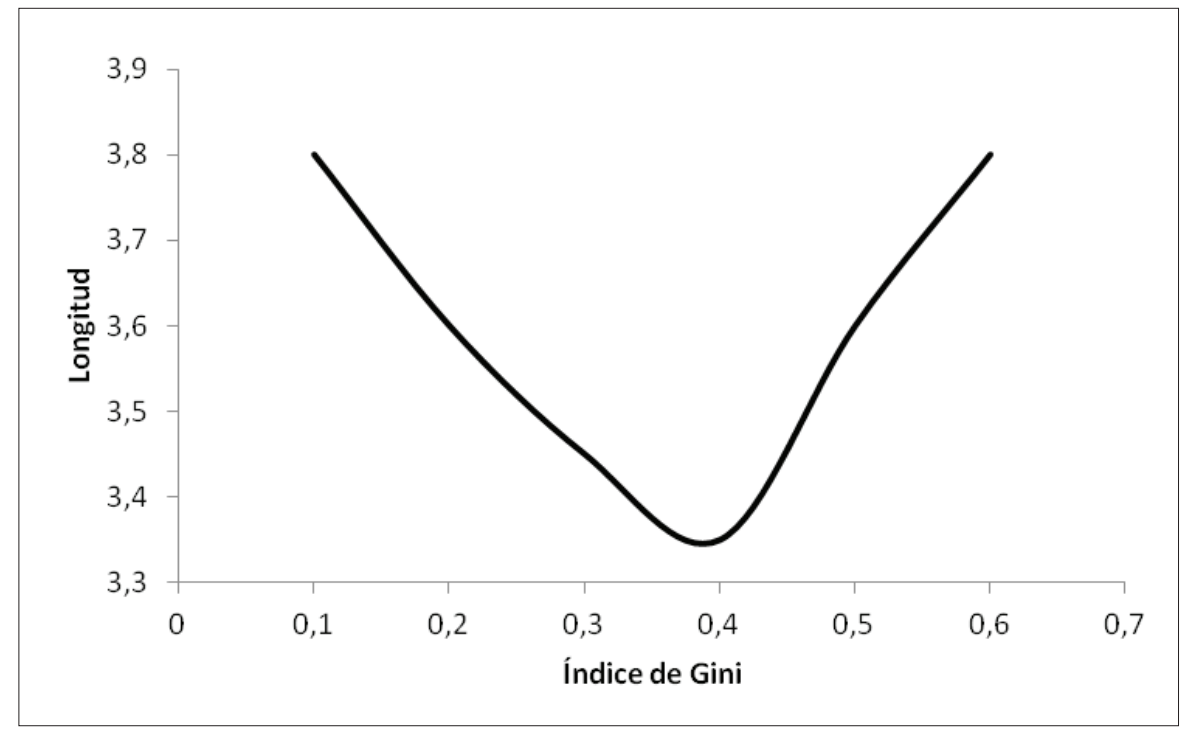

Fuente: Elaboración propia.

En resumen, la cuarta tanda de experimentos pone de manifiesto la existencia de un valor óptimo del índice de Gini de la distribución de vínculos que genera las mayores oportunidades para obtener empleo. Redes bastante igualitarias o desigualitarias en la distribución de vínculos son menos eficientes en la difusión de la información, y, por consiguiente, generan menos oportunidades de encontrar empleo que redes con grados de desigualdad intermedios para los mismos valores de densidad.

\section{CONCLUSIONES}

Los resultados de estos experimentos virtuales con redes artificiales sugieren que tanto el tipo de estructura de las redes sociales (regular, aleatoria, mundo pequeño y escala libre) como ciertas propiedades estructurales de dichas redes (densidad de red, índice de globalización de vínculos y grado de desigualdad en la distribución de vínculos) influyen significativamente en el acceso de los individuos al mundo laboral (un recurso de capital social inserto en tales redes). Asimismo, tales resultados apoyan la tesis de la 
relevancia de la estructura de las redes y de sus propiedades en los procesos dinámicos que tienen lugar en el interior de dichas redes (Barabási 2002; Burt 2004 y 2005; Coleman, Katz y Menzel 1957; Hedström 2005; Hedström y Bearman 2009; Granovetter 1973 y 1974; Milgram 1967; Newman 2003; Newman et al. 2003; Schelling 1978; Watts 2003).

Los resultados de estos experimentos virtuales ilustran de que lo importante para acceder al mercado laboral no es solo cuántos agentes están conectados entre sí (la densidad de red), sino también de cómo se distribuyen los vínculos entre los agentes (el índice de globalización de vínculos y el índice de Gini de la distribución de vínculos). Además, dichos resultados permiten evaluar la tesis de Granovetter (1973 y 1974) sobre la importancia de los vínculos débiles para el acceso de los individuos al mercado laboral bajo una nueva perspectiva. Tales experimentos muestran que se puede fomentar el acceso al mercado de trabajo sin tener necesariamente que elevar la densidad de red; es decir, la estructura en la distribución de vínculos de la red también afecta a la velocidad con que la información se difunde por la red, y, por consiguiente, al acceso al mercado de trabajo. Así pues, no solo la densidad influye en la velocidad de difusión de la información que fluye por las redes, como podría afirmar Granovetter, sino también la configuración en la distribución de dichos vínculos (Calvó-Armengol 2004 y 2006; González-Bailón 2006). Por lo tanto, la "fortaleza" (siguiendo la terminología de Granovetter) no solo recae en los vínculos débiles, sino también en la distribución de dichos vínculos débiles. Estos experimentos indican, pues, que aunque la tesis de Granovetter no es falsa, no llega a explorar del todo la compleja relación entre las estructuras de las redes sociales y el acceso de los individuos al mercado de trabajo.

La literatura sobre redes sociales ha señalado de diferentes maneras que el éxito de un individuo particular inserto en una red para llevar a cabo sus metas depende en parte del número de vínculos que posea (Coleman 1988; Lin 2001). De lo anterior, se puede derivar que el éxito de un individuo para acceder al mercado de trabajo depende en cierta medida del número de conexiones (en especial del número de vínculos débiles) que tenga en la red a la que pertenece. El dicho "no es solo lo que se sabe, sino a quién se conoce" sugiere que las redes sociales a las que pertenecen los individuos pueden ser tan importantes o más que los títulos que se posean a la hora de buscar un empleo (Erickson 2001). Por lo tanto, se puede concluir que la desigualdad en la distribución de vínculos entre los agentes de una red puede generar desigualdad en el acceso al mercado de trabajo. Por otro lado, se puede argumentar que la desigualdad social está relacionada con la desigualdad en la distribución de vínculos (Coleman 1988; Lin 2001), por lo que cuanto mayor sea la desigualdad social, mayor será la desigualdad en el acceso al mercado de trabajo.

Sin embargo, los resultados de estos experimentos ponen de manifiesto otra perspectiva diferente: redes desigualitarias con índices de Gini altos o redes igualitarias con índices de Gini bajos generan menos oportunidades de acceder a puestos de trabajo que redes con índices de Gini de valores intermedios. Es decir, dichos resultados sugieren que sociedades con niveles intermedios de desigualdad social son más proclives a 
generar mayores oportunidades para acceder al mercado de trabajo que sociedades igualitarias o muy desigualitarias.

Las políticas sociales de empleo deberían tener en cuenta no solo diferentes tipos de estructuras de redes sociales para aumentar la difusión de la información relevante para encontrar un empleo, sino también las propiedades estructurales de las redes sociales por donde fluye la información, como sugieren estos resultados. Sin embargo, el diseño de estructuras de redes muy eficientes en la difusión de la información debe estar sujeto no solo a criterios técnicos sino también a criterios normativos (González-Bailón 2006).

En esta investigación se ha estudiado cómo ciertas propiedades estructurales de las redes influyen en el acceso de los agentes al mercado de trabajo, pero también sería posible investigar otros aspectos estructurales. Se podría indagar, por ejemplo, cómo ciertas propiedades de gran trascendencia sociológica tales como el grado de intermediación, el grado de cercanía, los K-grupos o la velocidad con que se transmite la información por los vínculos fuertes frente a los débiles afectan a la transmisión de la información relevante para los individuos de una red, y, por consiguiente, al acceso al mercado laboral.

Se debe señalar, por último, que los experimentos virtuales poseen, como cualquier otra metodología de investigación social, algunos inconvenientes: en primer lugar, la comprobación empírica de los resultados de estos experimentos virtuales es muy costosa o irrealizable (el problema de la validación de los modelos basados en agentes), y si dichos resultados no se pueden comprobar empíricamente, entonces no hay manera de conocer su relevancia (Gilbert 2008; González-Bailón 2004). Sin embargo, dichos experimentos virtuales pueden abrir nuevos caminos de investigación al sugerir diferentes hipótesis empíricas y variadas cuestiones teóricas, como se ha tratado de mostrar a lo largo del artículo. En segundo lugar, otro inconveniente es la necesidad de comprobar si los resultados de la simulación se deben a causas substantivas o a defectos en la programación (el problema de la verificación de los modelos basados en agentes) (González-Bailón 2004). No obstante, este problema se puede paliar en parte si otros equipos científicos escriben sus propios programas y reproducen dichos experimentos para comprobar, como suele suceder con cualquier experimento científico, si los resultados son consistentes (Gilbert 2008).

\section{Referencias BibliográfícAs}

Allison, P. D. 1978. "Measures of Inequality". American Sociological Review 43:865-880.

Axelrod, R. 1997. "Advancing the Art of Simulation in the Social Science". Pp. 21-40 en Simulation Social Phenomena, coordinado por R. Conte, R. Hegselmann y P. Terna. Berlin: Springer-Verlag.

Barabási, A. L. 2002. Linked. The New Science of Networks. Cambridge, MA: Perseus.

Barabási, A. L. y R. Albert. 1999. "Emergence of Scaling in Random Networks". Science 286:509-12. 
Barabási, A. L. y E. Bonabeau. 2003. "Redes sin Escala”. Investigación y Ciencia julio: 58-67.

Beaman, L. A. 2012. "Social Networks and the Dynamics of Labour Market Outcomes: Evidence from Refugees Resettled in the U.S". Review of Economic Studies 79:128-161.

Boccaletti, S., V. Latora, Y. Moreno, M. Chávez y D. U. Hwang. 2006. “Complex networks: Structure and dynamics". Physics Reports 424:175-308.

Bourdieu, P. 1986. "The forms of Capital". Pp. 240-268 en Handbook of theory and research for the sociology of education, coordinado por J. G. Richardson. Nueva York: Greenwood.

Burt, R. S. 1992. Structure Holes: The Social Structure of Competition. Cambridge, MA: Harvard University Press.

Burt, R. S. 2004. "Structural holes and good ideas". American Journal of Sociology 110:349-99.

Burt, R. S. 2005. Brokerage and Closure. Oxford: Oxford University Press.

Calvó-Armengol, A. 2004. “Job Contact Networks”. Journal of Economic Theory 115:191-206.

Calvó-Armengol, A. 2006. Redes sociales y mercado de trabajo. Barcelona: El Centre de Recerca en Economia Internacional (CREI).

Calvó-Armengol, A. y M. O. Jackson 2004. "The Effects of Social Networks on Employment and Inequality". American Economic Review 94:426-454.

Calvó-Armengol, A. y M. O. Jackson 2007. "Networks in Labor Markets: Wage and Employment Dynamics and Inequality". Journal of Economic Theory 132:27-46.

Carrington, P. J., J. Scott y S. Wasserman. 2005. Models and Methods in Social Network Analysis. Cambridge: Cambridge University Press.

Coleman, J. S. 1988. "Social Capital in the Create of Human Capital". American Journal of Sociology 94:95-120.

Coleman, J. S. 1990. Foundations of Social Theory. Cambridge: Harvard University Press.

Coleman, J. S., H. Menzel y E. Katz. 1957. "The diffusion of an innovation among physicians". Sociometry 20:253-270.

Diestel, R. 2000. Graph Theory. Nueva York: Edición electrónica.

Epstein, J. M. 2006. Generative Social Science - Studies in Agent-Based Computational Modelling. Princeton: Princeton University Press.

Erirkson, B. H. 2001. "Good networks and Good Jobs: The value Social Capital to Employers and Employees". Pp. 127-158 en Social Capital: Theory and Research, coordinado por N. Lin, K. S. Cook, y R. S. Burt. Nueva York: Aldine de Gruyter.

Franzen, A. y D. Hangartner 2006. "Social Networks and Labour Market Outcomes: The Non-Monetary Benefits of Social Capital”. European Sociological Review 22:353-368.

García Faroldi, L. 2007. "Redes sociales y mercado de trabajo". REDES- Revista hispana para el análisis de redes sociales 13\#10. Consulta 1 de agosto de 2012 (http://revista-redes.rediris.es/ pdf-vol13/Vol13_10.pdf). 
García-Valdecasas, J. I. 2011a. "La Simulación Basada en Agentes: una nueva forma de explorar los fenómenos sociales". Revista Española de Investigaciones Sociológicas 136:91-110.

García-Valdecasas, J. I. 2011b. "Una definición estructural de capital social". REDES, Revista hispana para el análisis de redes sociales 20\#6. Consulta 1 de marzo de 2013 (http://revista-redes.rediris. es/pdf-vol20/vol20_6.pdf).

Gilbert, N. 2008. Agent-Based Models. Quantitative applications in the social science 153. Londres: Sage.

Gilbert, N. y A. Abbott. 2005. "Social Science Computation". American Journal of Sociology 110:859-863.

Gilbert, N. y K. G. Troitzsch 2005. Simulation for the Social Scientist. Milton Keynes: Open University Press.

González-Bailón, S. 2004. "¿Sociedades artificiales? Una introducción a la simulación social”. Revista Internacional de Sociología 39:199-222.

González-Bailón, S. 2006. "The Role of Dynamic Network in Social Capital: A Simulation Experiment". Papers: Revista de Sociología 80:171-194.

Granovetter, M. 1973. "The strength of weak ties". American Journal of Sociology 76:1360-1380.

Granovetter, M. 1974. Getting a Job. A Study of Contacts and Careers. Cambridge: Harvard University Pres.

Hanneman, R. A. y M. Riddle 2005. Introduction to Social Network Methods. Riverside, CA: University of California, Riverside.

Hedström, P. 2005. Dissecting the Social. Cambridge: Cambridge University Press.

Hedström, P. y P. Bearman eds. 2009. The Oxford Handbook of Analytical Sociology. Oxford: Oxford University Press.

Herreros, F. 2002. ¿Por qué confiar? El problema de la creación de capital social. Madrid: CEACS.

Hill, R. A. y R. I. M. Dunbar. 2003. "Social Network Size in Humans". Human Nature 14:53-72.

López-Roldan, P. y V. Alcaide. 2011. "El capital social y las redes personales en el estudio de las trayectorias laborales". REDES- Revista hispana para el análisis de redes sociales 20\#3. Consulta 1 de agosto de 2012 (http://revista-redes.rediris.es/pdf-vol20/vol20_3.pdf).

Lin, N. 2001. Social Capital. A Theory of Social Structure and Action. Cambridge: Cambridge University Press.

Macy, M. W. y A. Flache 2009. "Social Dynamics from the Bottom Up: Agent-Based Model of Social Interaction". Pp. 245-268 en The Oxford Handbook of Analytical Sociology, coordinado por P. Hedström y P. Bearman. Oxford: Oxford University Press.

Macy, M. W. y R. Miller 2002. "From Factors to Actors: Computational Sociology and Agent-Based Modelling". Annual Review of Sociology 28:143-66.

Milgram, S. 1967. "The Small World problem". Psychology Today 2:60-67.

Molina, J. L. 2001. El Análisis de Redes Sociales. Una Introducción. Barcelona: Edicions Bellaterra.

Montgomery, J. D. 1991. "Social Networks and Labor-Market Outcomes: Toward an Economic Analysis". The American Economic Review 81:1408-1418. 
Moody, J. 2009. "Network Dynamics". Pp. 247-274 en The Oxford Handbook of Analytical Sociology, coordinado por P. Hedström y P. Bearman. Oxford: Oxford University Press.

Newman, M. E. J. 2003. "The Structure and Function of Complex Networks". SIAM Review 45:167-256.

Newman, M. E. J., A. Barabási y D. J. Watts. 2003. The structure and Dynamics of Networks. Princeton: Princeton University Press.

Requena, F. 1989. "El concepto de Red Social". Revista Española de Investigaciones Sociológicas 48:137-152.

Requena, F. 1991. Redes sociales y mercado de trabajo. Elementos para una teoría del capital relacional. Madrid: Centro de Investigaciones Sociológicas, Colección Monografías 119.

Requena, F. ed. 2003. Análisis de Redes Sociales: Orígenes, Teorias y Aplicaciones. Madrid: Centro de Investigaciones Sociológicas.

Requena, F. 2006. "Redes sociales, mercado de trabajo y migraciones". Presentada en el $X$ Congreso de Inmigración: Globalización y movimientos transnacionales, 20-21-22 de abril de 2006, Almería.

Rodríguez, J. A. 1995. Análisis Estructural y de Redes. Madrid: Centro de Investigaciones Sociológicas.

Schelling, T. C. 1978. Micromotives and Macrobehavior. Nueva York: W. W. Norton.

Squazzoni, F. 2012. Agent-Based Computational Socioloy. Singapur: Wiley.

Toledo, F. y D. Bastourre 2006. "Capital social y recomposición laboral en Argentina. Un análisis para el período 1995-2000". Convergencia 13:141-171.

Wasserman, S. y K. Faust. 1994. Social Network Analysis. Cambridge: Cambridge University Press.

Watts, D. J. 1999a. "Network, Dynamic and Small-World Phenomenon". American Journal of Sociology 105:493-527.

Watts, D. J. 1999b. Small Worlds: The Dynamic between Order and Randomness. Princeton: Princeton University Press.

Watts, D. J. 2003. Six Degrees: The Science of a Connected Age. Nueva York: Norton.

Watts, D. J. y S. H. Strogatz 1998. "Collective Dynamics of "Small World" Networks". Nature 393:440-2.

Wilensky, U. 1999. NetLogo [Computer software]. Evanston, IL: Northwestern University, Center for Connected Learning and Computer-Based Modeling.

José Ignacio García-Valdecasas es Doctor en Sociología por la Universidad de Granada. Actualmente es investigador postdoctoral en la Universidad Carlos III de Madrid. Ha realizado estancias predoctorales en las universidad de Oxford y Leicester. Sus intereses investigadores se centran en la sociología analítica, simulación basada en agentes, análisis de redes sociales, teoría de juegos y sociología política.

RECIBIDO: $13 / 09 / 2012$

ACEPTADO: 28/03/2013

Publicado on-line: 27/02/2014 